\title{
In vivo, regulated reconstitution of spindle checkpoint arrest and silencing through chemical-induced dimerisation
}

Priya Amin, Sadhbh Soper Ní Chafraidh, loanna Leontiou and Kevin G. Hardwick*

Institute of Cell Biology, School of Biological Sciences, University of Edinburgh, King's Buildings, Max Born Crescent, Edinburgh, EH9 3BF, UK

* for correspondence:

Phone: 441316507091

Email: Kevin.Hardwick@ed.ac.uk

\section{Author contribution section:}

PA carried out the experiments and produced the data presented in Figs. 1, 2, S1 and S2.

SSNS carried out the experiments and produced the data presented in Figs. 3, 4, S3 and S4.

IL assisted with experiments, figure production and Fig. 4D model generation. 
bioRxiv preprint doi: https://doi.org/10.1101/406983; this version posted September 10, 2018. The copyright holder for this preprint (which was not certified by peer review) is the author/funder. All rights reserved. No reuse allowed without permission. 


\begin{abstract}
Chemical-induced dimerisation (CID) uses small molecules to control specific protein-protein interactions. Here, we employ CID dependent on the plant hormone abscisic acid (ABA) to reconstitute spindle checkpoint signalling in fission yeast. The spindle checkpoint signal usually originates at unattached or inappropriately attached kinetochores. These are complex, multiprotein structures with several important functions. To bypass kinetochore complexity, we take a reductionist approach to study checkpoint signalling. We generate a synthetic checkpoint arrest ectopically by inducing hetero-dimerisation of the checkpoint proteins Mph1 ${ }^{\text {Mps1 }}$ and Spc $7^{\text {KNL1 }}$. These proteins are engineered such that they can't localise to kinetochores, and only form a complex in the presence of $A B A$. Using this novel assay we are able to checkpoint arrest a synchronous population of cells within 30 minutes of ABA addition. This assay allows for detailed genetic dissection of checkpoint activation and importantly it also provides a valuable tool for studying checkpoint silencing. To analyse silencing of the checkpoint and the ensuing mitotic exit, we simply wash-out the ABA from arrested cells. We show here that silencing is critically-dependent on PP1 ${ }^{\text {Dis2 }}$ recruitment to Mph1 $1^{\mathrm{Mps} 1}-\mathrm{Spc} 7^{\mathrm{KNL} 1}$ signalling platforms.
\end{abstract}




\section{Introduction}

Spindle checkpoint signalling was initially reconstituted in Xenopus egg extracts (Kulukian et al., 2009; Minshull et al., 1994) and most recently using recombinant complexes of human checkpoint proteins (Faesen et al., 2017). Major advantages of such in vitro assays is that complex systems can be simplified through biochemical fractionation and manipulated through immunodepletion. They also enable the regulated addition of specific components, where the timing, concentration and activity of these can all be varied.

In parallel, yeast genetics drove identification of most of the molecular components of this pathway, the Mad and Bub proteins (Hoyt et al., 1991; Li and Murray, 1991), and their Cdc20 effector (Hwang et al., 1998; Kim et al., 1998). This combination of yeast genetics and in vitro reconstitution has proven invaluable when dissecting the molecular mechanism of action of spindle checkpoint signals and inhibition of the downstream effector Cdc20-APC/C (London and Biggins, 2014; Musacchio, 2015).

Here we have employed a hybrid approach, using yeast genetics and partial reconstitution of the pathway in vivo. We use synthetic biology to re-wire and simplify the upstream part of the checkpoint signalling pathway and chemical induced dimerisation (CID) to add an extra level of regulation that can be easily controlled experimentally in intact cells. Employing this strategy, we:

1) simplify the system, through regulated, ectopic activation of the spindle checkpoint, enabling kinetochore-independent studies.

2) use yeast genetics to enable rapid iterative analyses.

3) employ synthetic biology and CID to generate specific complexes in an experimentallycontrolled fashion.

4) use abscisic acid ( $A B A$ ) addition and wash-out to provide tight temporal control of the initiation and termination of checkpoint signalling.

More specifically, we generate a synthetic checkpoint arrest ectopically by inducing heterodimerisation of the checkpoint proteins $M p h 1^{\mathrm{Mps} 1}$ and Spc $7^{\mathrm{KNL} 1}$ in fission yeast. This leads to checkpoint arrest in a synchronous population of cells within 30 minutes of addition of the plant phytohormone abscisic acid (ABA). As expected this checkpoint response requires the downstream Mad and Bub factors. To analyse silencing of the checkpoint, we simply wash-out the $A B A$ from arrested cells and analyse mitotic exit. We find that the kinetics of release is critically-dependent on $\mathrm{PP} 1^{\text {Dis2 }}$ recruitment to the Mph1 $1^{\mathrm{Mps} 1}-\mathrm{Spc} 7^{\mathrm{KNL} 1}$ signalling platform. 


\section{Results}

We previously published a synthetic checkpoint arrest assay (SynCheck) where we activated the spindle checkpoint in fission yeast using heterodimers of TetR-Spc7 and TetR-Mph1 kinase (Yuan et al., 2017). However, in those experiments, dimerisation was constitutive, being driven by formation of Tet repressor dimers (TetR) and thus checkpoint signalling was challenging to regulate both in terms of initiation and termination. We controlled checkpoint arrest at the transcriptional level, using an $n m t$ promoter to drive expression of the TetR-Mph1 fusion protein. Unfortunately the fission yeast $n m t 1$ promoter requires induction in media lacking thiamine for several hours. As a consequence, the peak of arrest was observed $\sim 14$ hours after induction and wasn't as synchronous as one would wish. Here, to improve both timing and control, we have modified our approach by employing chemical-induced dimerisation (CID) to give us tight temporal control over the initiation and termination of checkpoint signalling.

\section{Generation of SynCheckABA}

Following the strategy of Crabtree and colleagues (Liang et al., 2011) we fused the PYL domain (residues 33 to 209) of the ABA receptor after the $\mathrm{N}$-terminal 666 amino acids of fission yeast $\mathrm{Spc} 7^{\mathrm{KNL} 1}$. By deleting the C-terminal half of $\mathrm{Spc} 7^{\mathrm{KNL} 1}$ this protein is unable to be targeted to kinetochores, as it lacks the Mis12-interacting region (Petrovic et al., 2016; Petrovic et al., 2014). This fusion protein was expressed from the constitutive adh21-promoter (Tanaka et al., 2009). The ABI domain (residues 126-423) of ABI1 was fused to the C-terminus of the Mph1 spindle checkpoint kinase. We also deleted the first 301 amino-acids of Mph1 to prevent it going to kinetochores (Heinrich et al., 2012). This Mph1-ABI fusion protein was expressed from the adh41-promoter (Tanaka et al., 2009). In the presence of abscisic acid the PYL and ABI domains are sufficient to form a tight complex (Miyazono et al., 2009), thus forming Mph1 $1^{A B I}$ Spc7 $7^{\mathrm{PYL}}$ complexes (Fig. 1A). We combined these constructs in a strain that also had the cdc2522 mutation, enabling synchronisation in G2, Bub1-GFP and mCherry-Atb2 to label microtubules.

\section{Inducing Spc7-Mph1 heterodimers to trigger a metaphase arrest}

Cells were synchronised in G2 using a temperature-sensitive cdc25-22 mutant which blocks cells in $\mathrm{G} 2$ after $3.5 \mathrm{~h}$ at $36^{\circ} \mathrm{C}$. When cells were shifted to $25^{\circ} \mathrm{C}$, they 'released' from the block enabling progression through the cell cycle. After 5 minutes, ABA was added to activate the spindle checkpoint through the formation of Spc7-PYL and Mph1-ABI heterodimers (Fig. 1B). We observed that over $70 \%$ of cells had short metaphase spindles 60 minutes after $A B A$ addition to the synchronous population of cells (Fig. 1C\&D). The metaphase arrest could be sustained for at least 4 hours (Fig. 1D). We tested a range of $A B A$ concentrations $(0-500 \mu M)$ and found that 
$250 \mu \mathrm{M}$ was optimal for reproducible, robust arrests (Fig. S1A). The ABA can be added later (eg. 20 mins after cdc25 release) and cells arrest with similar efficiency to that observed after antimicrotubule drug treatment (carbendazim, CBZ, see Fig. S1B). Without pre-synchronisation in G2, the mitotic index increases over time and reaches a peak four hours after ABA addition (Fig. S1C). In our previous SynCheck studies cells arrested for several hours but then died [10]. We wanted to determine whether the ABA arrest also had a significant affect on cell viability or whether our ability to release this arrest (through ABA wash-out) meant that viability was maintained. After ABA treatment we found a gradual drop in cell viability (see Fig. 2E), which was similar to that observed upon anti-microtubule drug treatment (data not shown). In the arrested cells, we observed Bub1 enrichment at the spindle poles (Fig. 1C). This is consistent with our previous SynCheck assay where movement of all spindle checkpoint proteins to spindle poles was reported to be Mad1-Cut7 kinesin driven (Yuan et al., 2017). As expected, deleting the first $\mathrm{N}$-terminal coiled coil (136 amino acids) of Mad1, required for its interaction with the Cut7 (Akera et al., 2015), prevented Bub1 accumulation at spindle poles. This de-localisation of checkpoint proteins from spindle poles did not affect the efficiency of the arrest (Fig. S1D), as was found in SynCheck (Yuan et al., 2017).

ABA-induced metaphase arrest is dependent on hetero-dimerisation of Spc7-PYL and Mph1$A B I$. Strains lacking either the Mph1-ABI component, or the Spc7-PYL component, failed to arrest in the presence of ABA (Fig. 1E). The ABA-induced arrest is checkpoint-dependent, as deleting the downstream checkpoint protein Mad1 abolished the arrest (Fig. 1E). In these constructs Spc7 and Mph1 lack their kinetochore-binding domains, making initiation of this arrest ectopic and independent of the complexities of the kinetochore. The Mph1-ABI, Spc7-PYL strain used above lacks endogenous mph1, which prevents all Mad/Bub checkpoint proteins from targeting to kinetochores (Heinrich et al., 2012). As an additional measure, to confirm kinetochore independence, we employed a strain containing the spc7-12A MELT mutant allele (Mora-Santos et al., 2016; Yamagishi et al., 2012). This mutant Spc7 kinetochore component cannot be phosphorylated by Mph1, preventing recruitment of Bub3-Bub1 and thereby Mad1Mad2 complexes to kinetochores. The spc7-12A mutant arrested with very similar efficiency to spc7+ cells under ABA control (Fig. S1F), arguing that the Spc7wt-PYL Mph1-ABI heterodimer does not need to be aided by endogenous kinetochore-based checkpoint signalling to generate a checkpoint arrest. Importantly, an spc7-12A-PYL fusion protein was unable to generate an arrest in combination with Mph1-ABI, demonstrating that the ectopic signaling scaffold does need to be phosphorylated on conserved Spc7 MELT motifs to recruit Bub3-Bub1 complexes for active signaling (Fig. S1G).

Critical outputs of checkpoint action are the stabilisation of cyclin B and securin. Using a modified strain we analysed cyclin B (Cdc13) levels in the ABA-induced arrest. Fig. 1F shows 
that Cdc13-GFP accumulated on short metaphase spindles and was enriched at mitotic spindle poles, as expected. As a technical aside, we have found that different tags can affect the efficiency of the ABA-induced arrest. For example, this Cdc13-GFP strain reproducibly arrests more efficiently than the strain containing Bub1-GFP (Fig. 1G). This is likely due to a partial loss of function when C-terminally tagging the Bub1 checkpoint protein. The Cdc13-GFP strain also contains the endogenous wild-type Mph1 gene, but we find that this does not significantly impact the efficiency of arrest (see Fig. S1E).

Thus we have reconstituted a robust, kinetochore-independent checkpoint arrest that can be initiated very simply in vivo through ABA addition to culture media. This works efficiently in both minimal (PMG) and rich (YES) fission yeast growth media. Hereafter, we refer to this assay as SynCheckABA.

\section{A novel spindle checkpoint silencing assay}

A significant advantage of SynCheckABA is the ability to reverse the effects of abscisic acid by simply washing cells with fresh media lacking $A B A$ and thereby releasing them from metaphase arrest (Fig. 2A/B). We can use this to study spindle checkpoint silencing, which has proven to be technically challenging in the past. Fig. $2 \mathrm{C} / \mathrm{D}$ demonstrates that when we wash-out the ABA we observe rapid cyclin degradation and spindle elongation (see also Fig. S2A).

\section{Regulation of spindle checkpoint silencing}

Previous work has shown that PP1 ${ }^{\text {Dis2 }}$ is a key spindle checkpoint silencing factor in yeasts (Meadows et al., 2011; Pinsky et al., 2009; Vanoosthuyse and Hardwick, 2009). The N-terminus of Spc $7^{\mathrm{KNL} 1}$ has two conserved motifs (SILK and RRVSF, also referred to as the A and $\mathrm{B}$ motifs) mediating stable PP1 ${ }^{\text {Dis2 }}$ association (Fig. $3 A$ ). Mutation of both binding sites leads to a lethal metaphase block in S. cerevisiae and in S. pombe (Meadows et al., 2011; Rosenberg et al., 2011). There are additional kinetochore binding sites for PP1 ${ }^{\text {Dis2 }}$ such as Klp5 and Klp6 (Meadows et al., 2011) and these are relevant to checkpoint silencing, although binding to Spc $7^{\mathrm{KNL} 1}$ appears to be the major player. In human cells similar motifs are found at the Nterminus of KNL1 and PP1 binding is regulated by Aurora B activity as this kinase can directly phosphorylate the B motif disrupting PP1 association (Liu et al., 2010).

Employing SynCheckABA, we tested mutations of the $A$ and $B$ motifs at the $\mathrm{N}$-terminus of $\mathrm{Spc} 7^{\mathrm{KNL} 1}$ and removal of the KIp5 and Klp6 kinesins. For the experiments below (Figs. 3 and 4), all strains contained endogenous, wild-type Mph1 kinase and thus are able to recruit checkpoint proteins to their kinetochores. This includes the Mph1 and Bub1 kinases which are also thought to also have 'error correction' functions. Thus silencing likely needs to take place not only at the ectopic Mph1 ${ }^{\text {Mps1 }}-S p c 7^{\text {KNL1 }}$ signalling scaffold, but also at kinetochores. Strains were presynchronised in G2 using cdc25, released and arrested at metaphase using ABA, and then 
washed to terminate checkpoint signalling. Progression through anaphase was then scored through the analysis of spindle elongation and/or cyclin B degradation (using Cdc13-GFP) over a 90 minute time-course. Mutation of the A-motif delayed spindle elongation by 30 minutes and the $A / B$ double mutant was delayed even more profoundly (Fig. 3B/C). This argues that PP1 activity on or near the Spc7 protein (previously phosphorylated by Mph1 kinase) is a limiting factor in checkpoint silencing. This system will prove useful for dissecting the regulation of PP1 binding to Spc7 in more detail, and for analysis of putative regulators of PP1 activity. Mutation of fission yeast kinesin 8 (either Klp5 or Klp6) leads to a stabilisation of microtubules, aberrant chromosome movements and long metaphase spindles (Gergely et al., 2016; Klemm et al., 2018; Meadows et al., 2011; West et al., 2002). In these mutants, checkpoint silencing defects can't simply be analysed through spindle elongation. Instead we imaged Cdc13-GFP and used the decrease in the number of cells with cyclin B enriched at their spindle poles as a measure of checkpoint silencing. Fig. 4A/B demonstrate that mutation of Klp6 significantly reduces the efficiency of silencing and cyclin B degradation. Finally, we analysed the silencing defect upon deletion of the PP1 ${ }^{\text {Dis2 }}$ phosphatase (dis24). In the dis2 $\Delta$ strain, which is rather sick, checkpoint silencing was extremely defective with no significant drop in Cdc13-GFP levels over the 90 minute time course (Fig. 4C). It should be noted that these dis2 4 strains display significant mitotic delays even in the absence of ABA addition, presumably because the lack of this mitotic phosphatase leads to pleiotropic mitotic defects (see Fig. S4 for images of these cells).

Thus, SynCheckABA neatly recapitulates the balance of opposing kinase and phosphatase activities between Mph1 ${ }^{\text {Mps1 }}$ dependent checkpoint activation and PP1-driven checkpoint silencing, on Spc7 $7^{\mathrm{KNL} 1}$ and Kinesin 8 dependent pathways (see general model in Fig. 4D).

\section{Discussion}

Here we have employed chemical-induced dimerisation to generate a rapid, controlled spindle checkpoint arrest: addition of abscisic acid (ABA) to SynCheckABA strains induces the heterodimerisation of $\mathrm{Mph} 1^{\mathrm{Mps} 1}-\mathrm{ABI}$ and $\mathrm{Spc} 7^{\mathrm{KNL} 1}-\mathrm{PYL}$ fusion proteins and this is sufficient to generate an activated signalling scaffold and metaphase arrest within minutes. Like our original SynCheck assay, which was driven by constitutive TetR homodimers (Yuan et al., 2017), this arrest acts independent of spindle checkpoint signalling at endogenous kinetochores, but is dependent on downstream checkpoint components such as Mad1.

A significant advantage of SynCheckABA is that we can wash out the ABA and study the kinetics and mechanism of spindle checkpoint silencing. This was not possible with the original 
SynCheck strain as we were unable to control TetR dimerisation and thus unable to dissociate the Mph1 ${ }^{\mathrm{Mps} 1}$-TetR-Spc $7^{\mathrm{KNL} 1}$-TetR signalling scaffold.

Using this new assay we have confirmed that protein phosphatase 1 (PP1 ${ }^{\text {Dis2 }}$ ) is critical for silencing the Mph1 ${ }^{\mathrm{Mps} 1}-\mathrm{Spc} 7^{\mathrm{KNL} 1}$ scaffold (Figs. 3-4). PP1 ${ }^{\mathrm{Dis} 2}$ binds to the $\mathrm{N}$-terminus of $\mathrm{Spc} 7^{\mathrm{KNL} 1}$, not far from the conserved MELT motifs that, once phosphorylated by Mph1 ${ }^{\mathrm{Mps} 1}$, will bind Bub3-Bub1 complexes to initiate MCC generation (Shepperd et al., 2012). Thus Spc7 $7^{\mathrm{KNL} 1}$ acts as the platform for both checkpoint activation and silencing and appears to be a major site of action of both checkpoint activation kinases and silencing phosphatases (Meadows et al., 2011). It is important to note that not all aspects of silencing are recapitulated in our ectopic assay, as some of these will relate to specific kinetochore processes that will not be captured here.

Kinesin 8 is also confirmed as a PP1 ${ }^{\text {Dis2 }}$ recruitment site relevant for checkpoint silencing in SynCheckABA. The phenotypes of the klp6 4 mutant suggests that targeting of PP1 to spindle microtubules and kinetochores is also relevant to mitotic exit from an ABA-induced arrest, even though the arrest is initiated away from the kinetochore (see Fig. 4D).

\section{Advantages of SynCheckABA, over other forms of reconstitution:}

We believe that all forms of spindle checkpoint reconstitution are useful for mechanistic dissection of this dynamic signalling pathway, whether this be in vitro within cytoplasmic extracts (Minshull et al., 1994), in vitro with purified recombinant proteins (Faesen et al., 2017) or in vivo with synthetically re-wired and simplified signalling pathways (SynCheckABA). The advantages of the latter system are:

1) the signalling pathway downstream of $S p c 7^{\mathrm{KNL} 1}$ and the downstream effectors are present at normal physiological levels and there are simple, quantitative physiological read-outs (cyclin B degradation, sister chromatid separation and/or anaphase spindle elongation).

2) checkpoint arrest is induced in the absence of additional stresses: simple addition of abscisic acid (low toxicity) to the growth media is sufficient for checkpoint activation. There is no need for a cold-shock (to depolymerise tubulin, nda3 arrest), heat-shock (to perturb temperature-sensitive kinetochore mutants), or overexpression of checkpoint activators.

3) The PYL and ABI domains have limited cross-reaction in yeast as they are derived from plant proteins. Although we haven't compared them directly, we believe that abscisic acid has certain advantages over the use of rapamycin, a very popular CID. To use rapamycin in fission yeast one needs to engineer strains to remove endogenous rapamycin-binding proteins, such as by deleting the fkh1+ gene which encodes a native FKBP12 domain (Ding et al., 2014). Importantly, because ABA doesn't bind tightly to the PYL domain, we can wash ABA out easily to initiate checkpoint silencing. In comparison, rapamycin is very difficult to wash-out making efficient release experiments unrealistic. 
4) compared to in vitro studies with large, recombinant complexes, these fission yeast experiments are simple, cheap and fast. The system also enables rapid iterative studies, due to ease of further genetic manipulation in yeast.

5) importantly, we can easily test candidate regulators (eg. silencing factors) without needing to know what complexes they are part of, purifying them and worrying about their relevant concentration and post-translational modifications.

6) compared to our transcriptionally controlled SynCheck (which employs nmt-tetR-Mph1) the $\mathrm{ABI}-\mathrm{PYL}$ system is less leaky, enabling sick strains such as the dis2 mutant analysed in Fig. 4 to be constructed. Previously, we were unable to isolate nmt-tetR-Mph1, dis2- strains, due to leaky expression from the weak nmt81 promoter.

Our ongoing studies with SynCheckABA will enable a detailed mechanistic dissection of PP1mediated spindle checkpoint silencing in fission yeast. We believe that ABA holds a lot of promise as an alternative CID to rapamycin and indeed that it has significant advantages.

\section{Acknowledgements}

We would like to thank Patrick Heun and Eftychia Kyriacou for providing constructs containing the abscisic acid binding hetero-dimerisation domains of PYL and ABI; Jonathan Millar for the PP1 binding spc7 mutants and the klp5,6 mutants; and Ken Sawin for the mCherry-Atb2 strain. This work was supported by a Seed Award from the Wellcome Trust to K.G.H. (108105) and the Wellcome Centre for Cell Biology core grant (092076). P.A. was supported by the Medical Research Council (MR/K501293/1), S.S.N.C. by the Wellcome Trust (105258) and I.L. by the Darwin Trust of Edinburgh. 


\section{Figure Legends}

Figure 1. Rapid induction of spindle checkpoint arrest using abscisic acid for chemicalinduced dimerisation of Mph1 $1^{\mathrm{Mps} 1}-\mathrm{Spc} 7^{\mathrm{KNL} 1}$.

(A) Schematic representation of the Mph1 ${ }^{\mathrm{Mps} 1}-\mathrm{Spc} 7^{\mathrm{KNL} 1}$ heterodimer induced by ABA addition.

(B) Work flow of the pre-synchronisation in G2 (cdc25-22), followed by release into mitosis at $25^{\circ} \mathrm{C}$ and then induction of checkpoint arrest through the addition of ABA.

(C) Fixed cell images taken of the arrested ABA-induced strain 60 minutes after ABA addition. Microtubules are seen in red mCherry-Atb2), the checkpoint protein in green (Bub1-GFP) and chromatin is stained with DAPI. Scale bar is $10 \mu \mathrm{m}$.

(D) Quantitation of cultures (+/- ABA addition) through a 4 hour time course after release from G2. Samples were fixed every 60 minutes and scored as metaphase arrested if they had short metaphase spindles and a single mass of condensed chromatin. $>100$ cells were analysed per strain at each time point. This experiment was repeated 3 times. Data plotted as mean $+/-$ SD.

(E) Quantitation of the strains indicated at the 60 minute time point after release from the G2 block (ABA added 5 mins after release). mad1 $\Delta$ is the Mph1-ABI Spc7-PYL strain with mad1 deleted. Cells were scored as metaphase arrested if they had short metaphase spindles and a single mass of condensed chromatin. 100 cells were analysed per strain at each time point. This experiment was repeated 3 times for each strain. Data plotted as mean +/- SD.

(F) Fixed cell images taken of the SynCheckABA strain with Cdc13-GFP at spindle poles bodies 60 minutes after ABA addition. Microtubules are seen in red (mCherry-Atb2 is labeled fission yeast tubulin), cyclin B in green (Cdc13-GFP) and chromatin is stained with DAPI. Scale bar is $10 \mu \mathrm{m}$.

(G) Quantitation comparing an ABA-induced metaphase arrest at 60 minutes between an Mph1ABI Spc7-PYL strain containing Bub1-GFP and another Cdc13-GFP. This experiment was repeated 2 times. Data plotted as mean $+/-S D$.

Figure 2. Silencing of spindle checkpoint signalling after abscisic acid wash-out.

(A) Schematic representation of the dissociation of $M p h 1^{\mathrm{Mps} 1}-\mathrm{Spc} 7^{\mathrm{KNL} 1}$ heterodimers after ABA wash-out.

(B) Silencing work flow: pre-synchronisation in G2 (cdc25-22), induction of checkpoint arrest through the addition of $A B A$, and subsequent wash-out of $A B A 60$ minutes later.

(C) Fixed cell images taken of the arrested SynCheckABA strain at 0, 15, 25 and 35 minutes after ABA wash-out. Microtubules are seen in red (mCherry-Atb2), cyclin Bin green (Cdc13-GFP) and chromatin is stained with DAPI. Scale bar is $10 \mu \mathrm{m}$. See Fig. S2B for an alternatively coloured version of similar images. 
(D) Quantitation of Cdc13-GFP at spindle pole bodies in the SynCheckABA cultures (+ ABA/DMSO). Samples were fixed and scored for the presence of Cdc13 at spindle pole bodies. The +DMSO control didn't arrest in metaphase. $>150$ cells were analysed per strain at each time point. This experiment was repeated 3 times. Data are plotted as mean +/- SD.

(E) The viability of SynCheckABA arrested strains was determined by plating cells $0,60,120$, 180 and 240 minutes after release from a G2 block where DMSO or ABA was added 5 minutes after release from the $\mathrm{G} 2$ block. Cell viability over time was plotted as a percentage relative to that at time zero. Cells were plated in triplicate. The experiment was repeated 3 times and data are plotted as mean $+/-$ SD.

Figure 3. Checkpoint silencing in SynCheckABA is dramatically slowed when the Spc $7^{\text {KNL1 }}$ binding sites for PP1 ${ }^{\text {Dis2 }}$ are deleted.

(A) Schematic of Spc $7^{\mathrm{KNL} 1}$ indicating the N-terminal PP1 binding motifs (A motif: SILK and B motif: RRVSF). The MELT motifs form binding sites for Bub3-Bub1 complexes once they have been phosphorylated by Mph1 kinase.

(B) Images of cells expressing wild-type Spc7 $7_{1-666}$ or mutants with deletion of the A motif or both the $A$ and $B$ motifs. Time points were analysed at the time of ABA washout (time zero) and 30 and 60 minutes post-wash. Scale bar is $10 \mu \mathrm{m}$. See Fig. S3 for non red-green colour scheme. (C) Quantitation of this release from checkpoint arrest in the strains expressing wild-type Spc7 ${ }_{1}$ 666 or mutants with deletion of the A motif or deletion of both the A and B motifs. This experiment was repeated three times. $>100$ cells were analysed per strain at each time point. Data are plotted as mean +/- SD.

(D) Schematic model of SynCheckABA: activating (Mph1) and silencing (PP1) factors bind nearby on the Spc7 scaffold. The balance of their activities will determine how much MCC is generated and thus whether anaphase onset is inhibited.

Figure 4. Checkpoint silencing in SynCheckABA is also slowed when other recruitment sites for PP1 ${ }^{\text {Dis2 }}$ are removed from spindles.

(A) Deletion of the kinesin 8, Klp6, leads to reduced silencing efficiency. Images of cells with and without Klp6 deleted are shown after ABA washout (time zero) and 30, 60 and 90 minutes post-wash. Microtubules are seen in red (mCherry-Atb2), cyclin Bin green (Cdc13-GFP) and chromatin is stained with DAPI. Scale bar is $10 \mu \mathrm{m}$.

(B) Quantitation of this release from checkpoint arrest in strains with and without Klp6. Cells were scored as arrested if Cdc13-GFP was enriched at spindle poles. This experiment was repeated three times. $>100$ cells were analysed per strain at each time point. Data plotted as mean $+/-$ SD.

(C) dis2- mutants have profound silencing defects. Quantitation of the release from the 
checkpoint arrest is shown for wild-type and dis2- cells (+ABA/DMSO). Cells were scored as metaphase arrested if they had short metaphase spindles and a single mass of condensed chromatin. DMSO controls illustrate that dis2- cells are generally sick, but that ABA addition induces the SynCheckABA, resulting in elevated levels of metaphase arrested cells. This arrest persists for $>60 \mathrm{~m}$ after ABA washout as dis2- cells struggle to silence the checkpoint. This experiment was repeated three times. $>200$ cells were analysed per strain at each time point. Data plotted as mean +/- SD. See Fig. S4 for fixed cell images from this timecourse.

(D) General model of relevant PP1-dependent silencing pathways. This schematic describes two pools of PP1 $1^{\text {Dis2 }}$ one that is recruited to the ectopic Spc7-Mph1 signalling scaffold, via the A and $\mathrm{B}$ motifs on Spc7, and a second pool that is more generally recruited to the spindle through interaction with kinesin 8 (Klp6). These two pools act together to inhibit MCC-APC/C assembly and thereby enable checkpoint silencing and mitotic exit.

Four Supplementary Figures and a fission yeast strain list (Supplementary Table 1) are also associated with this article online. 


\section{Materials and Methods}

$P_{\text {adh } 41}-m p h 1_{303-678}-3 \times H A-A B I$

Mph1303-678 was amplified from a pDONR 201 plasmid containing Mph1303-678 (Yuan et al., 2017).

$3 \times$ HA was amplified from a plasmid from the Allshire lab containing codon optimised PYL-3xHA. ABI was amplified from a PMT_CID_ABI_VS_H vector from the Heun lab. These PCR fragments were Dpn1 treated and assembled into a Sma1-digested and antarctic phosphatase treated, gel purified pRad41 yeast expression vector by Gibson assembly.

\section{Padh21-Spc71-666-PYL}

A yeast expression vector (plY03 (Yuan et al., 2017)) was digested with Nhe1 and Xho1 and gel purified. The insert (mCherry-3xFlag-Spc71-666) was used as a template to amplify Spc71-666. PYL was amplified from a bVNI-221 vector from the Heun lab. The fragments were then assembled into the digested plY03 vector backbone using Gibson assembly.

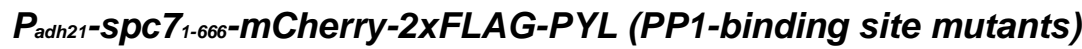

Plasmids containing full length Spc7 PP1-binding mutants $(\triangle \mathrm{A}$ : deletion of residues 136-150; $\triangle \mathrm{AB}$ : deleting residues $136-150$ and residues 331-345) (Millar lab) were used as templates to amplify mutant versions of Spc71-666. Nhel-NLS and Pacl sites were introduced during amplification, allowing Spc7 constructs to be digested and ligated into digested pIY03-derived vector backbone which also contained a C-terminal mCherry-2Xflag-PYL tag.

\section{Fission yeast strains}

These are listed in Supplementary Table 1.

\section{cdc25-22 synchronisation}

Cells were grown at $25^{\circ} \mathrm{C}$ for $1-2$ days on YES (rich yeast media, with additional leucine, arginine, lysine, histidine and uracil) plates. They were then pre-cultured in $10 \mathrm{ml}$ of liquid YES containing amino acid supplements at $25^{\circ}$ over the day and inoculated into a larger culture of YES overnight. The following day, log phase cultures were shifted to $36^{\circ} \mathrm{C}$ for 3.5 hours to block in G2. After this, cultures were cooled quickly in iced water to rapidly shift them back to $25^{\circ} \mathrm{C}$ and release them from the $\mathrm{G} 2$ block.

\section{Synthetic arrest assay}

Following a cdc25-22 block, 250mM abscisic acid stock (Sigma Aldrich A1049) was added to cultures 5 minutes after release (20 minutes if comparing to a carbendazim arrest) to achieve a final concentration of $250 \mathrm{uM}$ (unless otherwise stated).

\section{Synthetic arrest assay wash-out}


Following an ABA-induced synthetic arrest, the cells were washed 3 times (with $50 \mathrm{ml}$ YES).

\section{Fixing cells and microscopy}

1-1.5 $\mathrm{ml}$ of culture was centrifuged for 1 minute at 6000 RPM. The cell pellet was fixed in 200$500 \mathrm{ul} 100 \%$ ice-cold methanol. To image cells, 8 ul of the cell-methanol suspension was added to a glass slide, when the methanol evaporated, $1-2 \mathrm{ul} \mathrm{DAPI}(0.4 \mathrm{ug} / \mathrm{ml})$ was added to the sample and a glass cover slip was placed on top.

Cells were imaged immediately using a 100x oil immersion lens and a Zeiss Axiovert 200M microscope (Carl Zeiss Ltd.), equipped with a CoolSnap CCD camera (Photometrics) and Slidebook 5.0 software (3i, Intelligent Imaging Innovations, Inc.). Typical acquisition settings: 300 ms exposure (FITC \& TRITC), 100 ms exposure DAPI, 2x binning, Z-series over $3 \mu \mathrm{m}$ range in $0.5 \mu \mathrm{m}$ steps (7 planes).

\section{Carbendazim arrest}

Following a cdc25-22 block, $3.75 \mathrm{mg} / \mathrm{ml}$ of carbendazim was added to cultures 20 minutes after release to achieve a final concentration of $100 \mathrm{ug} / \mathrm{ml}$.

\section{Cell viability assay}

Following a synthetic arrest assay, cells from $1 \mathrm{ml}$ of culture were harvested by centrifugation at 6000 RPM for 1 minute and re-suspended in $1 \mathrm{ml}$ of distilled water. 10-fold serial dilutions were made in distilled water. $0.1 \mathrm{ml}$ of cells diluted by factors of 100 and 1000 were plated in triplicate. Colony forming units per $\mathrm{ml}$ of culture was calculated and cell viability over time was plotted as a percentage relative to that at time zero. 


\section{References}

Akera, T., Goto, Y., Sato, M., Yamamoto, M., and Watanabe, Y. (2015). Mad1 promotes chromosome congression by anchoring a kinesin motor to the kinetochore. Nat Cell Biol 17, 1124-1133.

Ding, L., Laor, D., Weisman, R., and Forsburg, S.L. (2014). Rapid regulation of nuclear proteins by rapamycin-induced translocation in fission yeast. Yeast 31, 253-264.

Faesen, A.C., Thanasoula, M., Maffini, S., Breit, C., Muller, F., van Gerwen, S., Bange, T., and Musacchio, A. (2017). Basis of catalytic assembly of the mitotic checkpoint complex. Nature 542, 498-502.

Gergely, Z.R., Crapo, A., Hough, L.E., McIntosh, J.R., and Betterton, M.D. (2016). Kinesin-8 effects on mitotic microtubule dynamics contribute to spindle function in fission yeast. Mol Biol Cell 27, 3490-3514.

Heinrich, S., Windecker, H., Hustedt, N., and Hauf, S. (2012). Mph1 kinetochore localization is crucial and upstream in the hierarchy of spindle assembly checkpoint protein recruitment to kinetochores. J Cell Sci 125, 4720-4727.

Hoyt, M.A., Totis, L., and Roberts, B.T. (1991). S. cerevisiae genes required for cell cycle arrest in response to loss of microtubule function. Cell $66,507-517$.

Hwang, L.H., Lau, L.F., Smith, D.L., Mistrot, C.A., Hardwick, K.G., Hwang, E.S., Amon, A., and Murray, A.W. (1998). Budding yeast Cdc20: A target of the spindle checkpoint. Science 279, 1041-1044.

Kim, S.H., Lin, D.P., Matsumoto, S., Kitazono, A., and Matsumoto, T. (1998). Fission yeast Slp1: an effector of the Mad2-dependent spindle checkpoint. Science 279, 1045-1047.

Klemm, A.H., Bosilj, A., Glunc ic, M., Pavin, N., and Tolic, I.M. (2018). Metaphase kinetochore movements are regulated by kinesin- 8 motors and microtubule dynamic instability. Mol Biol Cell 29, $1332-1345$.

Kulukian, A., Han, J.S., and Cleveland, D.W. (2009). Unattached kinetochores catalyze production of an anaphase inhibitor that requires a Mad2 template to prime Cdc20 for BubR1 binding. Dev Cell 16, 105-117.

$\mathrm{Li}, \mathrm{R}$., and Murray, A.W. (1991). Feedback control of mitosis in budding yeast. Cell 66, 519-531. Liang, F.S., Ho, W.Q., and Crabtree, G.R. (2011). Engineering the ABA plant stress pathway for regulation of induced proximity. Sci Signal 4, rs2.

Liu, D., Vleugel, M., Backer, C.B., Hori, T., Fukagawa, T., Cheeseman, I.M., and Lampson, M.A. (2010). Regulated targeting of protein phosphatase 1 to the outer kinetochore by KNL1 opposes Aurora B kinase. J Cell Biol 188, 809-820.

London, N., and Biggins, S. (2014). Signalling dynamics in the spindle checkpoint response. Nat Rev Mol Cell Biol 15, 736-748.

Meadows, J.C., Shepperd, L.A., Vanoosthuyse, V., Lancaster, T.C., Sochaj, A.M., Buttrick, G.J., Hardwick, K.G., and Millar, J.B. (2011). Spindle Checkpoint Silencing Requires Association of PP1 to Both Spc7 and Kinesin-8 Motors. Dev Cell 20, 739-750.

Minshull, J., Sun, H., Tonks, N.K., and Murray, A.W. (1994). A MAP kinase-dependent spindle assembly checkpoint in Xenopus egg extracts. Cell 79, 475-486.

Miyazono, K., Miyakawa, T., Sawano, Y., Kubota, K., Kang, H.J., Asano, A., Miyauchi, Y., Takahashi, M., Zhi, Y., Fujita, Y., et al. (2009). Structural basis of abscisic acid signalling. Nature 462, 609-614.

Mora-Santos, M.D., Hervas-Aguilar, A., Sewart, K., Lancaster, T.C., Meadows, J.C., and Millar, J.B. (2016). Bub3-Bub1 Binding to Spc7/KNL1 Toggles the Spindle Checkpoint Switch by Licensing the Interaction of Bub1 with Mad1-Mad2. Curr Biol 26, 2642-2650.

Musacchio, A. (2015). The Molecular Biology of Spindle Assembly Checkpoint Signaling Dynamics. Curr Biol 25, R1002-1018.

Petrovic, A., Keller, J., Liu, Y., Overlack, K., John, J., Dimitrova, Y.N., Jenni, S., van Gerwen, S., Stege, P., Wohlgemuth, S., et al. (2016). Structure of the MIS12 Complex and Molecular Basis of Its Interaction with CENP-C at Human Kinetochores. Cell 167, 1028-1040 e1015. 
Petrovic, A., Mosalaganti, S., Keller, J., Mattiuzzo, M., Overlack, K., Krenn, V., De Antoni, A., Wohlgemuth, S., Cecatiello, V., Pasqualato, S., et al. (2014). Modular assembly of RWD domains on the Mis12 complex underlies outer kinetochore organization. Mol Cell 53, 591-605. Pinsky, B.A., Nelson, C.R., and Biggins, S. (2009). Protein phosphatase 1 regulates exit from the spindle checkpoint in budding yeast. Curr Biol 19, 1182-1187.

Rosenberg, J.S., Cross, F.R., and Funabiki, H. (2011). KNL1/Spc105 Recruits PP1 to Silence the Spindle Assembly Checkpoint. Curr Biol 21, 942-947.

Shepperd, L.A., Meadows, J.C., Sochaj, A.M., Lancaster, T.C., Zou, J., Buttrick, G.J., Rappsilber, J., Hardwick, K.G., and Millar, J.B. (2012). Phosphodependent Recruitment of Bub1 and Bub3 to Spc7/KNL1 by Mph1 Kinase Maintains the Spindle Checkpoint. Curr Biol 22, 891899.

Tanaka, K., Chang, H.L., Kagami, A., and Watanabe, Y. (2009). CENP-C functions as a scaffold for effectors with essential kinetochore functions in mitosis and meiosis. Dev Cell 17, 334-343. Vanoosthuyse, V., and Hardwick, K.G. (2009). A novel protein phosphatase 1-dependent spindle checkpoint silencing mechanism. Curr Biol 19, 1176-1181.

West, R.R., Malmstrom, T., and McIntosh, J.R. (2002). Kinesins klp5(+) and klp6(+) are required for normal chromosome movement in mitosis. J Cell Sci 115, 931-940.

Yamagishi, Y., Yang, C.H., Tanno, Y., and Watanabe, Y. (2012). MPS1/Mph1 phosphorylates the kinetochore protein KNL1/Spc7 to recruit SAC components. Nat Cell Biol 14, 746-752.

Yuan, I., Leontiou, I., Amin, P., May, K.M., Soper Ni Chafraidh, S., Zlamalova, E., and Hardwick, K.G. (2017). Generation of a Spindle Checkpoint Arrest from Synthetic Signaling Assemblies.

Curr Biol 27, 137-143. 
A

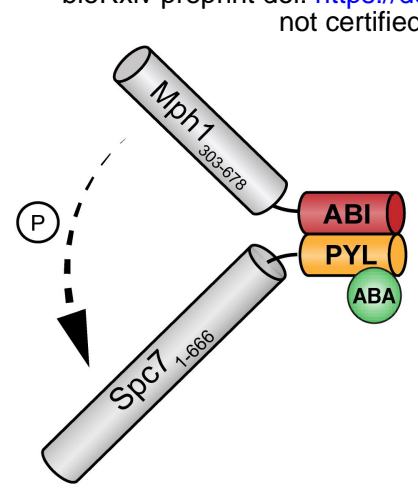

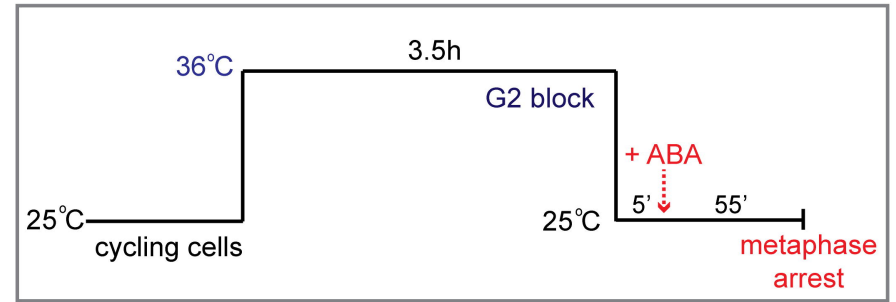

arrest

C

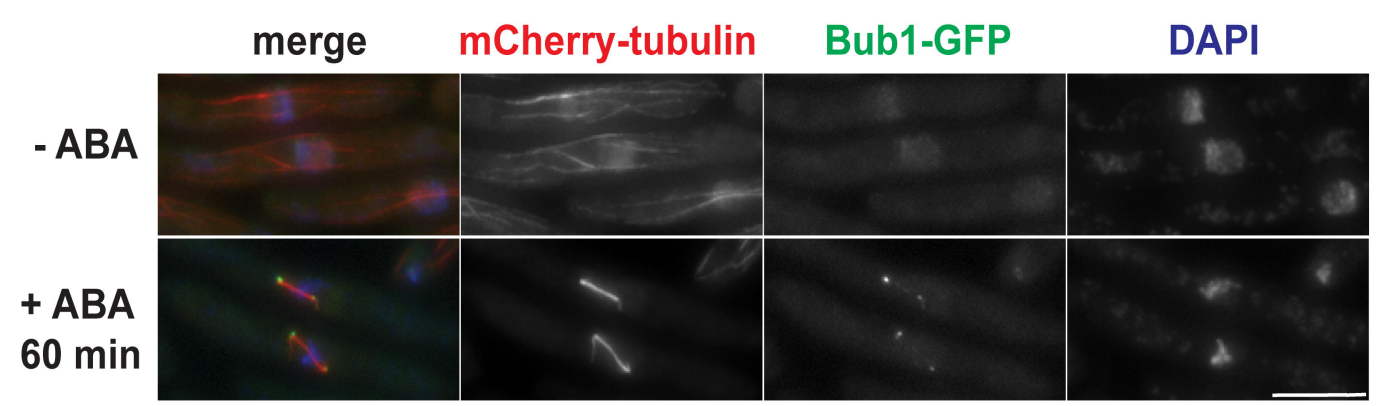

adh21-spc7(1-666)-PYL, adh41-mph1(303-678)-3xHA-ABI, mph1 $\Delta, c d c 25-22$
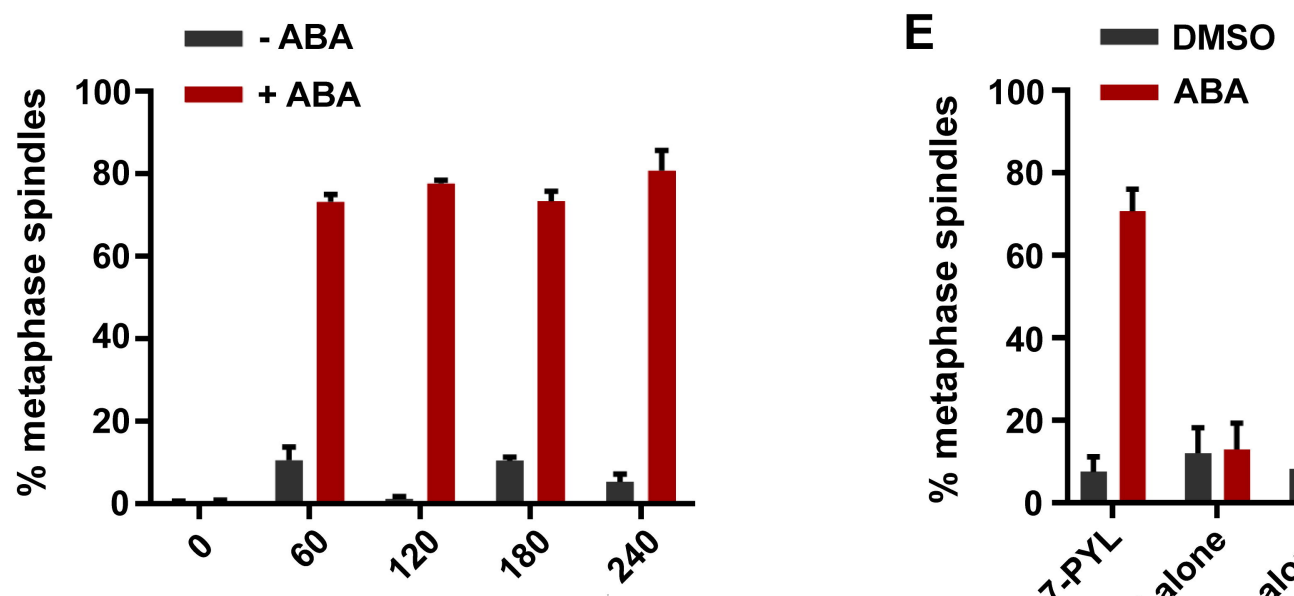

time (mins) after $\mathbf{G} 2$ release

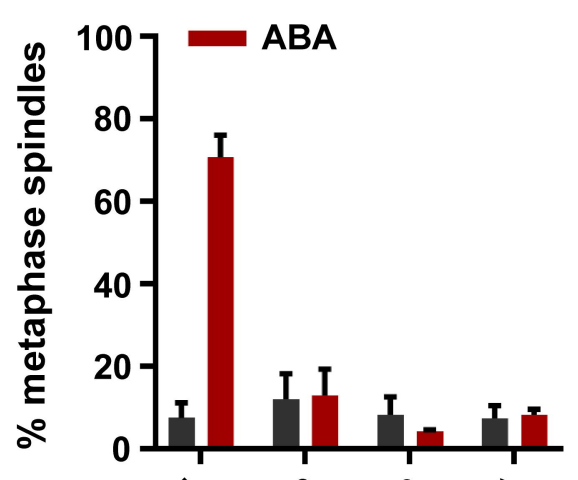

$F$

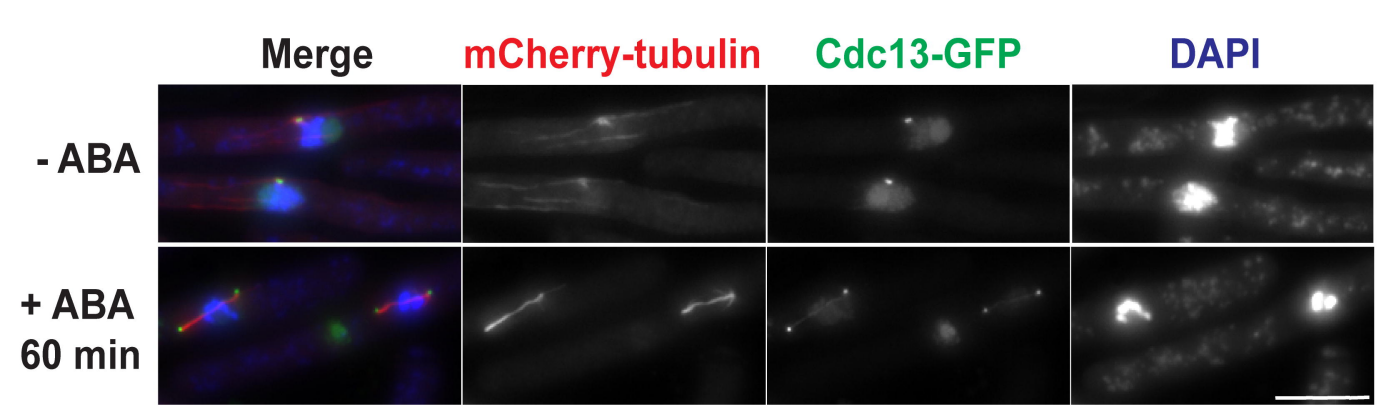

adh21-spc7(1-666)-PYL, adh41-mph1(303-678)-3xHA-ABI, cdc25-22

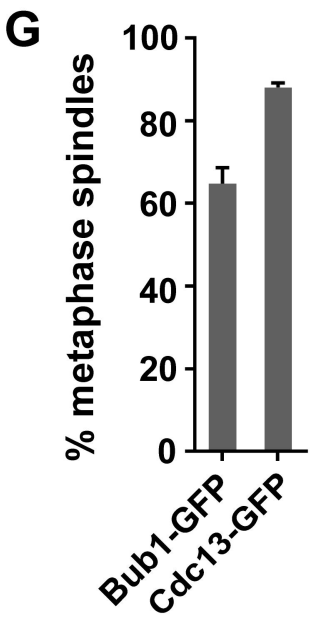

Figure 1 Amin et al 


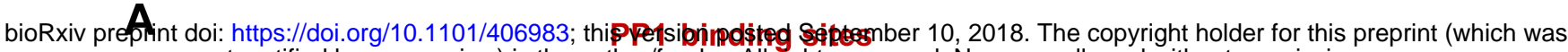
not certified by peer review) is the author/funder. All rights reserved. No reuse allowed without permission.

$\mathrm{Spc7}_{1-666}$ MELT motifs

B

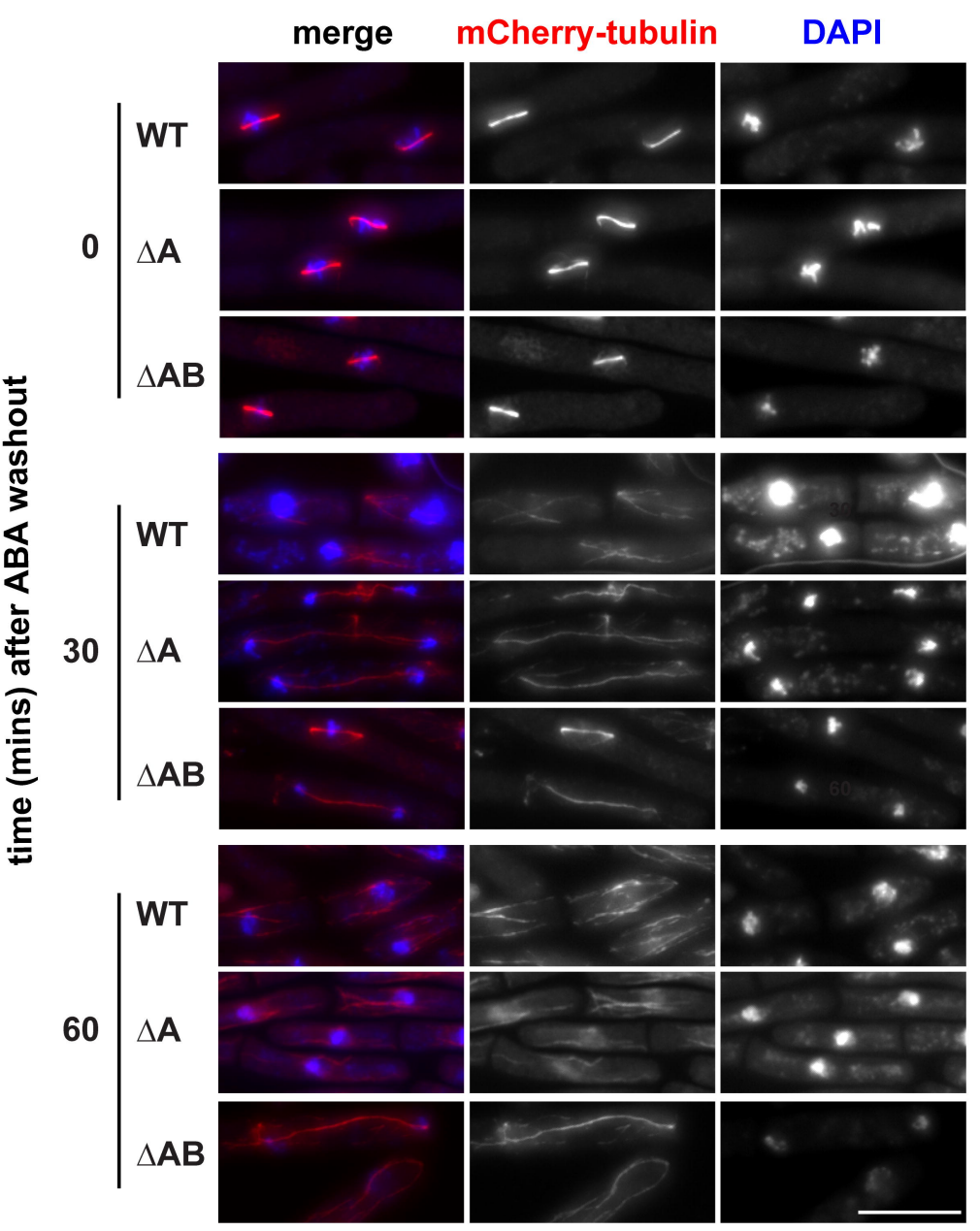

C

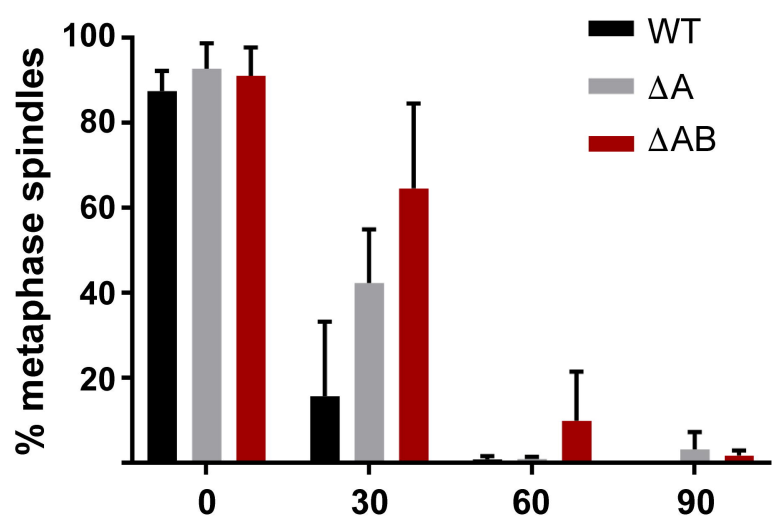

time (mins) after ABA washout

adh21-spc7(1-666)mutants-mCherry-2xFLAG-PYL adh41-mph1(303-678)-3xHA-ABI cdc25-22

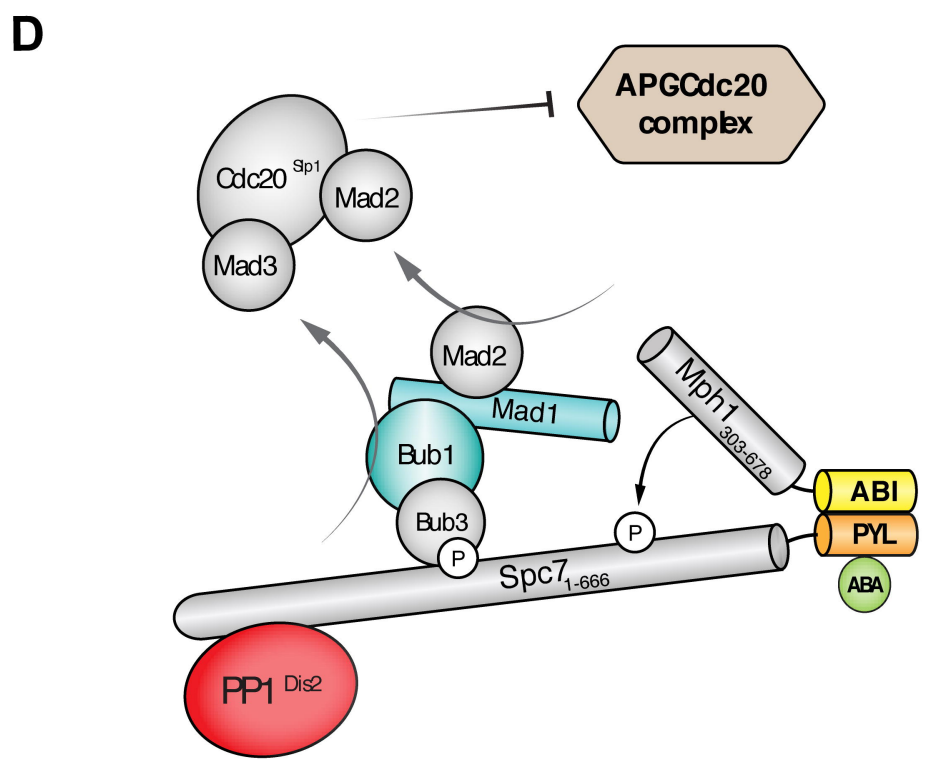

Figure 3 Amin et al 
LRxiv preprint doi: https://doi.org/10.1101/406983; this version posted September 10,2018. The copyright holder for this preprint (which was

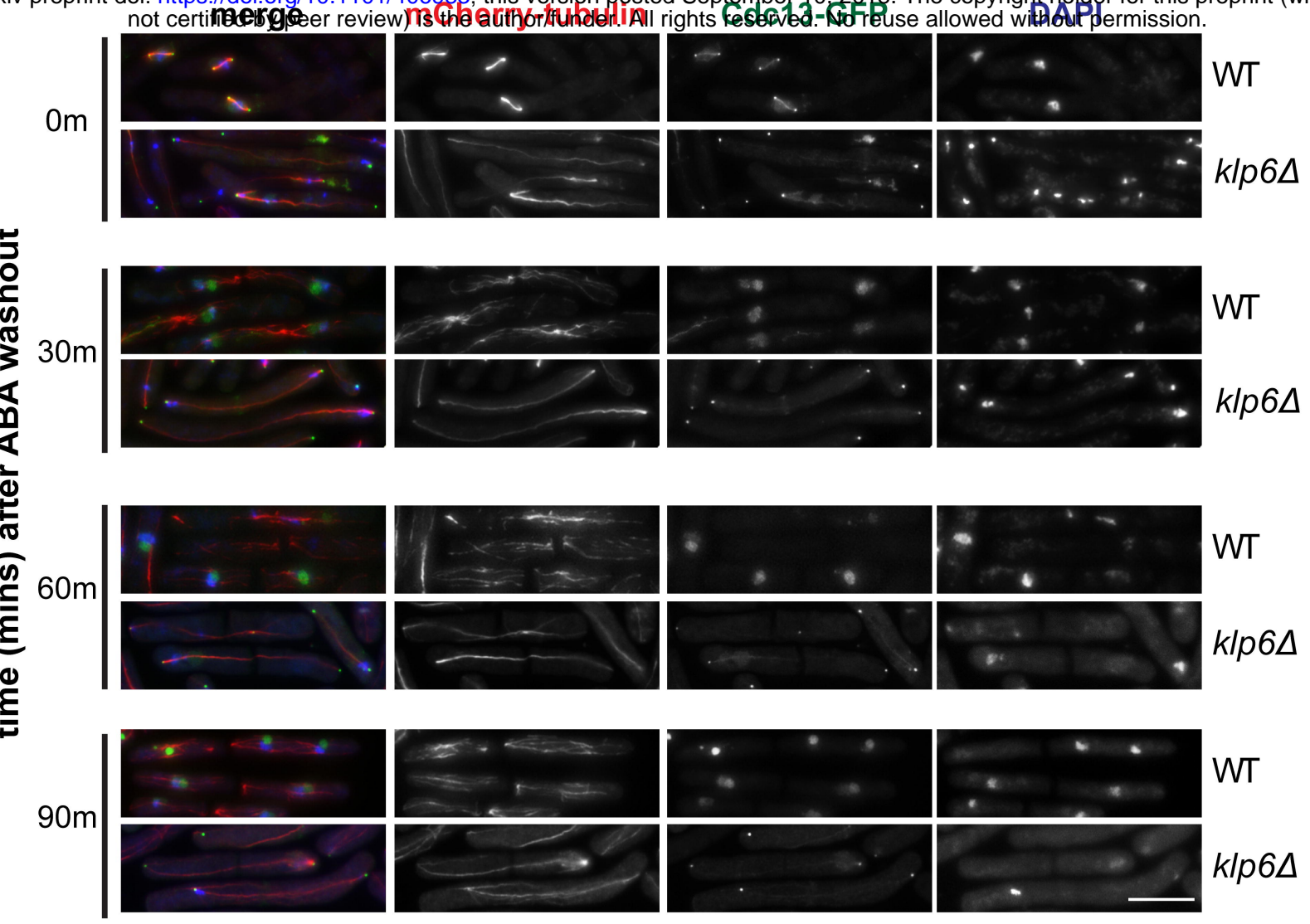

B

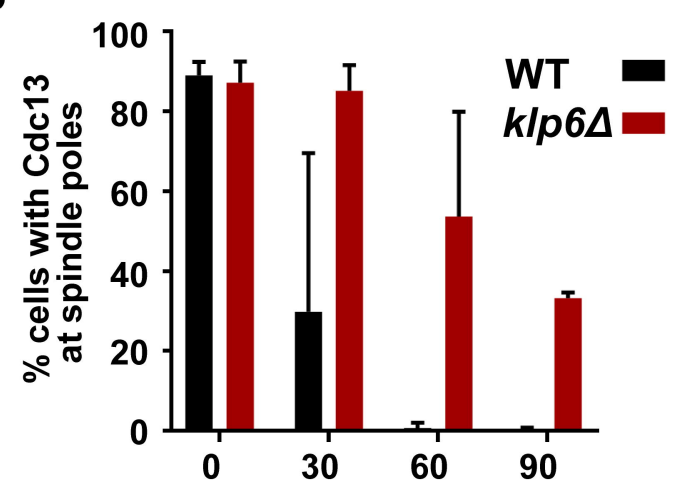

time (mins) after ABA washout
C

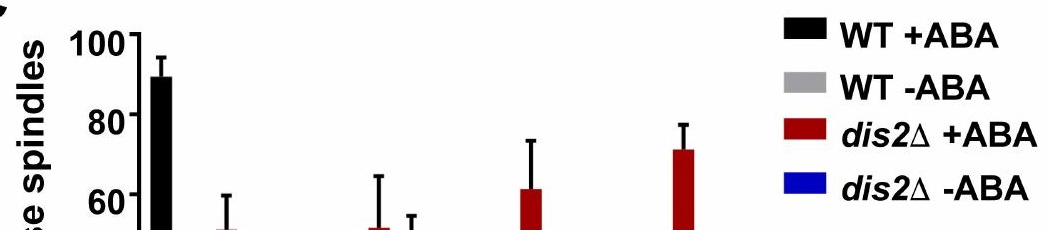

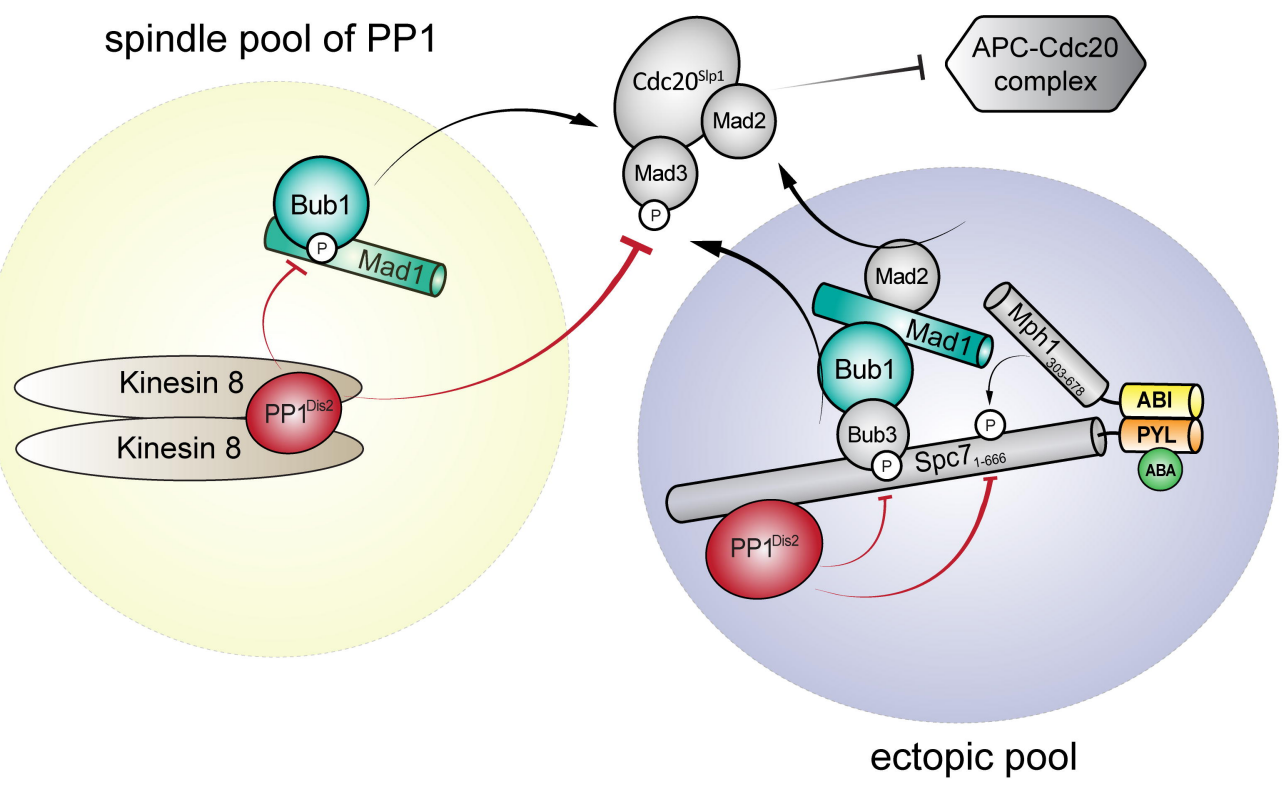

Figure 4 Amin et al 